\title{
DOSE-RELATED EFFECTS OF SMALLPOX VACCINE
}

\author{
Sharon E. Frey, M.D., Frances K. Newman, M.S., John Cruz, B.S., W. Brian Shelton, Ph.D., \\ Janice M. Tennant, M.P.H., Tamara Polach, B.S., Alan L. Rothman, M.D., Jeffrey S. Kennedy, M.D., \\ Mark Wolff, Ph.D., Robert B. Belshe, M.D., and Francis A. Ennis, M.D.
}

\begin{abstract}
Background We conducted a double-blind, randomized trial of three dilutions of vaccinia virus vaccine in previously unimmunized adults in order to assess the clinical success rates, humoral responses, and virus-specific activity of cytotoxic T cells and interferon- $\gamma$-producing T cells.

Methods Sixty healthy adults were inoculated intradermally by bifurcated needle with undiluted vaccine (dose, $10^{7.8}$ plaque-forming units [pfu] per milliliter), a 1:10 dilution (dose, $10^{6.5}$ pfu per milliliter), or a 1:100 dilution (dose, $10^{5.0}$ pfu per milliliter); there were 20 subjects in each group. The subjects were monitored with respect to vesicle formation (an indicator of successful vaccination), the viral titer at the time of peak lesion formation, antiviral antibodies, and cellular immune responses.
\end{abstract}

Results A vaccinia vesicle developed in 19 of the 20 subjects who received undiluted vaccine (95 percent), 14 of the 20 who received the 1:10 dilution (70 percent), and 3 of the 20 who received the 1:100 dilution (15 percent). One month after vaccination, 34 of 36 subjects with vesicles had antibody responses, as compared with only 1 of 24 subjects without clinical evidence of vaccinia virus replication. Vigorous cytotoxic T-cell and interferon- $\gamma$ responses occurred in 94 percent of subjects with vesicles, and a cytotoxic T-cell response occurred in only one subject without a vesicle.

Conclusions The vaccinia virus vaccine (which was produced in 1982 or earlier) still has substantial potency when administered by a bifurcated needle to previously unvaccinated adults. Diluting the vaccine reduces the rate of successful vaccination. The development of vesicular skin lesions after vaccination correlates with the induction of the antibody and T-cell responses that are considered essential for clearing vaccinia virus infections. (N Engl J Med 2002;346:1275-80.)

Copyright $\odot 2002$ Massachusetts Medical Society.

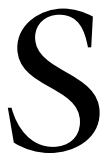
MALLPOX vaccine (vaccinia virus) is highly effective in immunizing against smallpox and can prevent disease when given as late as two to three days after exposure. ${ }^{1} \mathrm{~A}$ comprehensive public health program coupled with vaccination enabled the World Health Assembly to declare the world free of smallpox in $1980 .{ }^{2}$ General use of the vaccine in the United States ended in 1972. Currently, less than half the world's population has been exposed either to smallpox (variola virus) or to the vaccine. ${ }^{3}$ This fact prompted several government and world health authorities to warn about the serious threat of smallpox as a biologic weapon. ${ }^{3,4}$ The probability of a release of smallpox is not known, but the effect could be catastrophic in an unimmunized population. $^{5}$

The immune responses required to protect a person from smallpox after vaccination are not completely understood. Inadvertent vaccination resulted in severe complications and death from vaccinia in children with a T-cell deficiency and an adult with undiagnosed infection with human immunodeficiency virus type 1 (HIV-1) but not in children with agammaglobulinemia. ${ }^{6-8}$ Therefore, detailed analyses of T-cell responses in addition to antibody responses after vaccination may improve our understanding of the effects of dilution on the immunogenicity of vaccinia virus vaccine.

The last lots of vaccinia vaccine manufactured in the United States, in 1982, were produced by the classic method involving scarification of calves followed by collection of vaccinia virus from draining calf lymph. Concern about contamination with bacteria or other agents from bovines makes further use of this method unfeasible. The lyophilized vaccine, which is stored at the Centers for Disease Control and Prevention (CDC) in Atlanta, contains approximately $10^{8}$ pockforming units per milliliter. Supplies of lyophilized vaccine in the United States consist of an estimated 15 million doses. In addition, 70 to 90 million doses of frozen liquid-formulation smallpox vaccine have been identified in long-term storage by a vaccine manufacturer (Aventis, Swiftwater, Pa.); the U.S. government is reportedly negotiating to acquire this vaccine. Efforts are under way to develop a tissue-culturederived vaccinia virus vaccine. ${ }^{9}$ In the interim, we evaluated the available vaccine to determine whether dilution altered the rate of successful viral replication at the inoculation site and immune responses.

From the Department of Medicine, National Institute of Allergy and Infectious Diseases Vaccine and Treatment Evaluation Unit, Saint Louis University School of Medicine, St. Louis (S.E.F., F.K.N., W.B.S., J.M.T., T.P., R.B.B.); the Center for Infectious Disease and Vaccine Research, University of Massachusetts Medical School, Worcester (J.C., A.L.R., J.S.K., F.A.E); and the Emmes Corporation, Rockville, Md. (M.W.). Address reprint requests to Dr. Frey at the Division of Infectious Diseases and Immunology, Saint Louis University Health Sciences Center, 3635 Vista Ave. (FDT-8N), St. Louis, MO 63110.

This article was published at www.nejm.org on March 28, 2002. 


\section{METHODS}

\section{Vaccine}

The vaccine (Dryvax, Wyeth Laboratories, Marietta, Pa.; lot no. 4998391 [titer, $10^{7.7}$ plaque-forming units, or pfu, per milliliter] and lot no. 4008257 [titer, $10^{7.8}$ pfu per milliliter]) and diluent were provided by the CDC. The vaccine is a lyophilized product prepared from calf lymph. The diluent contains 50 percent glycerin, 0.25 percent phenol, and 0.005 percent brilliant green dye in water. The vaccine was reconstituted on the day of administration according to the package insert. The undiluted vaccine was serially diluted with diluent. Coded vials were stored at $4^{\circ} \mathrm{C}$ until use. Because the vaccine was highly viscous and difficult to manipulate, viral titers were determined in each of the 19 vials prepared, as described previously. ${ }^{10}$ Titers are expressed as the number of plaqueforming units per milliliter.

\section{Study Design and Subjects}

The study was a randomized, double-blind trial conducted at the National Institute of Allergy and Infectious Diseases Vaccine and Treatment Evaluation Unit in St. Louis. The protocol was approved by the institutional review board of Saint Louis University. All subjects provided written informed consent and were enrolled between April 2000 and October 2000. Long-term follow-up to assess the duration of immune responses is ongoing.

Healthy adults 18 to 30 years of age were eligible if they had no vaccination scar; no history of vaccinia virus vaccination; normal renal and hepatic serum chemical values; negative tests for hepatitis B surface antigen, hepatitis C virus antibody, and rapid plasma reagin; and a negative HIV-l enzyme-linked immunosorbent assay (ELISA). Exclusion criteria included the contraindications against vaccination noted in the package insert (pregnancy, immunosuppression, and eczema), a history of vaccination with live attenuated virus within 60 days before the study, the receipt of blood products or immune globulin within 6 months before the study, and household contact, sexual contact, or occupational exposure to pregnant women, immunosuppressed persons, persons with eczema, or infants less than 12 months of age.

A total of 60 subjects were enrolled and were randomly assigned to receive undiluted vaccine, a 1:10 dilution of vaccine, or a 1:100 dilution of vaccine. Twenty subjects were enrolled in each group. Group assignment was revealed to the subjects after day 45. Laboratory personnel remained unaware of treatment assignments until all assays were completed. Subjects were inoculated by scarification: a bifurcated needle that held a drop of vaccine was pressed 15 times into the skin of the upper arm. Vaccination sites were covered with folded gauze and a semipermeable adhesive membrane (Tegaderm, 3M Health Care, St. Paul, Minn.) to avoid autoinoculation or exposure of personal contacts. Dressings were changed every three to five days until the lesion formed an eschar.

The primary end point was the rate of success of vaccination. Success was defined by the presence of a primary vesicle at the inoculation site seven to nine days after scarification. Other signs and symptoms of the replication of vaccinia virus include edema, tenderness, and erythema at the site of vaccination and regional lymphadenopathy. Subsequently, the vesicle (Fig. 1) evolves into a small ulcer over which a scab forms, ultimately leaving a small scar. The determination of successful vaccination was made by a single physician who was unaware of the subjects' treatment assignments. Secondary end points included measurement of antibody responses and cellular immune responses.

\section{Isolation of Virus and Antibody Assays}

Swab samples of each lesion were obtained on day 7, 8, or 9 after scarification and cultured on continuous African-green-monkey kidney cells (BSC- 40 cells), and the virus present in the swab samples was quantitated by a plaque assay as described previously. ${ }^{10}$ Neutralization assays were performed on serum samples collected just before vaccination (day 0 ) and one month and one year after vaccination; the end point was a 60 percent reduction in the number of plaques as described previously. ${ }^{11}$ Serum binding antibody levels were measured by ELISA as described previously.12 Vaccinia virus antigen and serum samples to be used as positive and negative controls were provided by Dr. George Ludwig (U.S. Army Medical Research Institute of Infectious Diseases, Fort Detrick, Md.).

\section{Cytotoxicity Assays}

Cryopreserved peripheral-blood mononuclear cells obtained from all subjects on day 0 and at six months were thawed and tested in the same assay. Target cells were autologous Epstein-Barr virus-transformed lymphoblastoid cells that had been infected with vaccinia virus one day earlier and that were labeled with chromium-51 on the day of the assay.13,14 Effector cells were exposed to virus-infected autologous peripheral-blood mononuclear cells for six days at $37^{\circ} \mathrm{C}$ and then added at various effector-target ratios (10:1, 30:1, and 90:1) in 96-well U-bottom plates, in triplicate, for 4.5 hours as described previously. ${ }^{14,15}$ At each effector-target ratio, vaccinia-specific immune lysis was calculated as the difference between the percent lysis of infected targets and the percent lysis of uninfected targets. The number of effector cells required to lyse 30 percent of target cells (referred to as lytic units) per million cells was determined by an exponential-fit method ${ }^{16}$ with the use of commercial software (Proteins International, Rochester Hills, Mich.).

\section{Interferon- $\gamma$ Assays}

A modified enzyme-linked immunospot assay was used to detect live virus-specific release of interferon- $\gamma$ by cryopreserved peripheral-blood mononuclear cells as previously described, ${ }^{16}$ except that stock vaccinia virus was used to stimulate peripheral-blood mononuclear cells at a multiplicity of infection of 1.0 pfu per cell. The frequency of interferon- $\gamma$-positive $T$ cells specific for vaccinia virus per million peripheral-blood mononuclear cells was determined, and the results were considered positive if the number of spots per million peripheral-blood mononuclear cells in virusstimulated wells was twice as high as the number of spots per million cells in the control wells and if at least 20 spots per million peripheral-blood mononuclear cells were present. Preliminary studies (Ennis FA: unpublished data) indicated that most of the $\mathrm{T}$ cells that release vaccinia virus - specific interferon- $\gamma$ are CD8+.

\section{Proliferation Assays}

Proliferation assays were performed in replicates of five as described previously. ${ }^{14,15,17}$ For each group of five replicates, the stimulation index was calculated as the mean of the three intermediate values (expressed as counts per minute).

\section{Statistical Analysis}

The objective of this study was to assess the effects of diluted vaccinia virus vaccine on clinical and laboratory indicators of protective immunity. To be considered successful, a given dilution had to evoke a response in at least 18 of the 20 subjects in a group (a success rate of 90 percent). Thus, a sample size of 20 involved an 8 percent chance of rejecting a vaccine with a 95 percent success rate and a 32 percent chance of rejecting a marginally acceptable vaccine with a 90 percent success rate. A sample size of 20 was selected on the basis of these considerations and the understanding that the selection of a dilution will not depend solely on the dichotomous measure of success or failure in any group. Pairs of success rates were compared with use of standard asymptotic methods for binomial comparisons, and all three groups were compared with use of the Kruskal-Wallis test for trend. ${ }^{18}$

T-cell responses were analyzed by analysis of variance with the use of SPSS statistical software (SPSS, Chicago). Multiple post hoc 


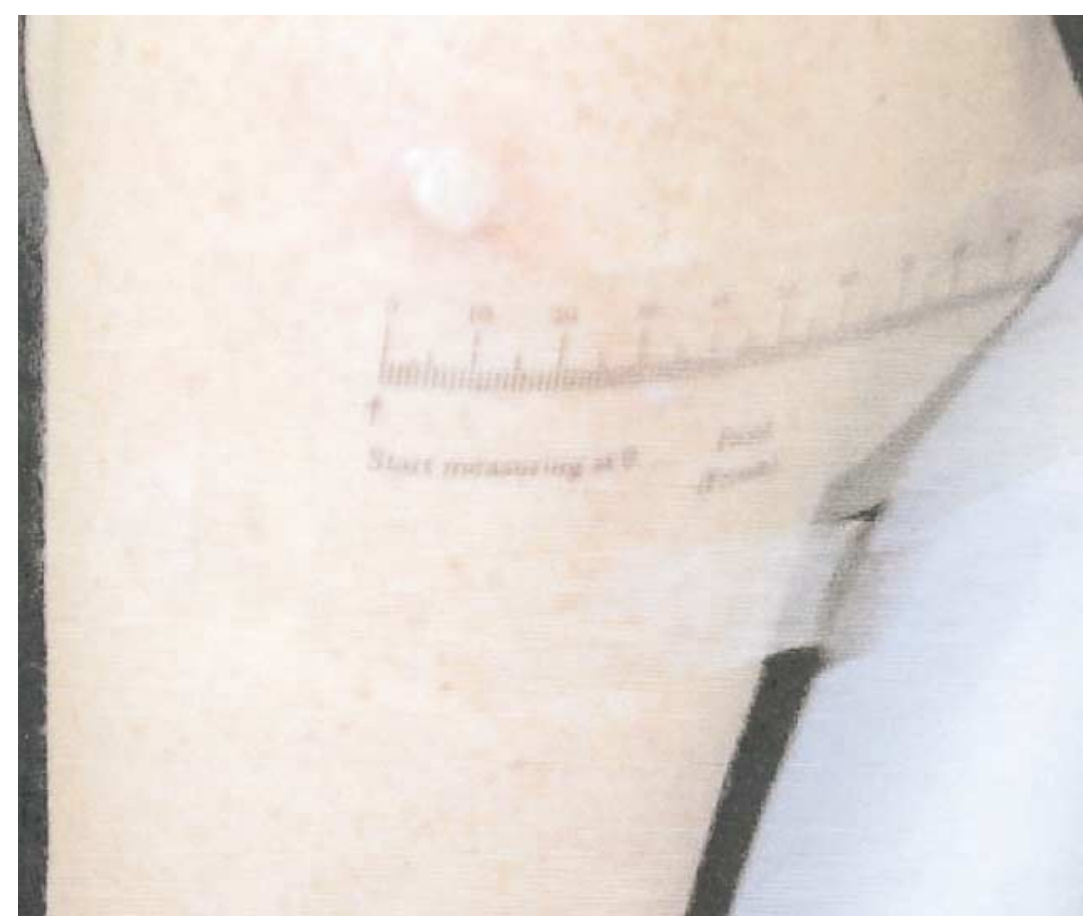

Figure 1. Typical Vesicle on the Upper Arm 10 Days after Scarification.

comparisons of the study groups were made with the use of the Tukey adjustment for multiplicity. All t-tests were two-sided.

\section{RESULTS}

\section{Clinical Findings}

Of the 60 subjects, 2 had a fever (temperature, up to $39^{\circ} \mathrm{C}\left[102.2^{\circ} \mathrm{F}\right]$ ) for 1 or 2 days beginning 11 and 12 days, respectively, after vaccination. One subject reported a stiff neck on days 12 and 13 after vaccination. One subject reported intermittent dizziness, floaters, and tachycardia starting on day 15 after vaccination and lasting two weeks. One subject had an intermittent rash on the arms and legs and muscle aches on days 9 through 13. Two subjects had an elevated alanine aminotransferase level $(\leqslant 84 \mathrm{U}$ per liter) on day 28; the elevation resolved two weeks later in one subject and was still present at the time of the last follow-up visit in the other. One subject had a transient, mild decrease in the hemoglobin level (13.4 to $11.7 \mathrm{~g}$ per deciliter) on day 28 . On urinalysis at six months, one subject transiently had 11 white cells per high-power field. Three subjects reported that the skin around the dressing was irritated. One subject in whom vaccination failed reported myalgias and pain at the vaccination site seven days after vaccination. There were no serious adverse events. One subject who received the 1:100 dilution of vaccine withdrew after vaccination for reasons unrelated to the study. Fifty-five of the subjects provided blood specimens at one year, as planned.

\section{Success Rates}

Vaccination success rates - defined by the formation of a vesicle at the inoculation site seven to nine days after vaccination - were dose-dependent $(\mathrm{P}<$ 0.001 by the Kruskal-Wallis test): vaccination was successful in 19 of 20 subjects who received undiluted vaccine (mean viral titer, $10^{7.8}$ pfu per milliliter; range, $10^{7.4}$ to $\left.10^{8.3}\right), 14$ of 20 who received a $1: 10$ dilution (mean viral titer, $10^{6.5}$ pfu per milliliter; range, $10^{6.1}$ to $\left.10^{7.0}\right)$, and 3 of 20 who received a 1:100 dilution (mean viral titer, $10^{5.0}$ pfu per milliliter; range, $10^{4.2}$ to $\left.10^{5.9}\right)$. The success rate was significantly lower in the group that received the $1: 10$ dilution than in the group that received the undiluted vaccine $(70$ percent vs. 95 percent; absolute difference, 25 percent; 95 percent confidence interval for the difference, 3 to 47 percent; $\mathrm{P}=0.03$ with the use of asymptotic methods). Once viral infection of the skin was initiated, the resulting lesion was approximately $1 \mathrm{~cm}$ in diameter, regardless of the dose of vaccine. Vaccinia virus was isolated from swab samples of skin lesions in 35 of the 36 subjects with vesicles. 
Table 1. Clinical Success Rates and Antibody Responses to the Various Doses of Vaccine in Subjects Who Had Not Previously Been Vaccinated. *

\begin{tabular}{|c|c|c|c|}
\hline VARIABLE & $\begin{array}{l}\text { UNDILUTED VACCINE } \\
\text { (DOSE, } 10^{7.8} \mathrm{pfu} / \mathrm{ml} \text { ) }\end{array}$ & $\begin{array}{l}\text { 1:10 DILUTION } \\
\text { (DOSE, } 10^{6.5} \mathrm{pfu} / \mathrm{ml} \text { ) }\end{array}$ & $\begin{array}{c}\text { 1:100 Dilution } \\
\text { (DOSE, } 10^{5.0} \mathrm{pfu} / \mathrm{ml} \text { ) }\end{array}$ \\
\hline Clinical success - no. of subjects/total no. (\%) & $19 / 20(95)$ & $14 / 20(70)$ & $3 / 20(15)$ \\
\hline Mean maximal size of resulting lesion $-\mathrm{mm}$ & 11.6 & 10.6 & 10.3 \\
\hline \multicolumn{4}{|l|}{ Serum neutralizing antibody titer } \\
\hline $\begin{array}{l}\text { Increase by a factor of } 4 \text { or more at } 1 \mathrm{mo} \\
- \text { no. of subjects/total no. } \dagger\end{array}$ & $17 / 19$ & $14 / 14$ & $3 / 3$ \\
\hline Measurable titer at $1 \mathrm{yr}-$ no. of subjects/no. tested & $15 / 17$ & $9 / 13$ & $3 / 3$ \\
\hline Reciprocal GMT at $1 \mathrm{mo}$ & 36 & 41 & 25 \\
\hline Reciprocal GMT at $1 \mathrm{yr}$ & 11 & 6 & 10 \\
\hline \multicolumn{4}{|l|}{ Serum antibody titer on ELISA } \\
\hline $\begin{array}{l}\text { Increase by a factor of } 4 \text { or more at } 1 \mathrm{mo} \\
- \text { no. of subjects/total no. } \dagger\end{array}$ & $14 / 19$ & $11 / 14$ & $2 / 3$ \\
\hline Measurable titer at $1 \mathrm{yr}-$ no. of subjects/no. tested & $17 / 17$ & $10 / 13$ & $3 / 3$ \\
\hline Reciprocal GMT at $1 \mathrm{mo}$ & 430 & 328 & 400 \\
\hline Reciprocal GMT at $\mathrm{l} \mathrm{yr}$ & 245 & 170 & 159 \\
\hline
\end{tabular}

${ }^{*}$ Clinical success was defined by the formation of a vesicle at the inoculation site seven to nine days after vaccination. The titer in each group is the mean value for 19 vials. Values shown for the geometric mean titer (GMT) after vaccination are the mean titers in subjects who had vesicles. ELISA denotes enzyme-linked immunosorbent assay.

$\nmid$ Data shown are for subjects with vesicles.

\section{Antibody Responses}

Among 36 subjects with vesicles, neutralizing antibody titers and antibody titers on ELISA increased by a factor of at least 4 at one month in 34 and 26 subjects, respectively (Table 1 ). In the 33 subjects with vesicles who returned for follow-up at one year, neutralization titers at one year averaged 23.7 percent of the titers on day 28 (95 percent confidence interval, 7 to 33 percent) and ELISA titers averaged 64.3 percent of the titers on day 28 (95 percent confidence interval, 44.8 to 96.6 percent) (Table 1 ).

\section{Cytotoxic T-Cell and Interferon- $\gamma$ Responses}

The development of a vesicle correlated with the development of cytotoxic T-cell responses in 31 of 32 subjects (of 4 other subjects with vesicles, 2 had nonviable cells and 2 had a high background response on the cytotoxic T-cell assay) and an increase in the number of $\mathrm{T}$ cells positive for virus-specific interferon- $\gamma$ by enzyme-linked immunospot assay in 31 of 34 subjects. Figure $2 \mathrm{~A}$ demonstrates vaccinia virusspecific cytotoxic T-cell activity six months after scarification with undiluted vaccine, the 1:10 dilution of vaccine, and the 1:100 dilution of vaccine. When the magnitude of the cytotoxic $\mathrm{T}$-cell responses was based on the percent lysis among subjects with vesicle formation in each group, there was no significant difference between the group given undiluted vaccine and the group given the $1: 10$ dilution $(\mathrm{P}=0.052)$, but the difference between the group given undiluted vaccine and the group given the 1:100 dilution was significant $(\mathrm{P}<0.001)$. None of the subjects had vac- cinia virus-specific cytotoxic T-cell activity on day 0 (before vaccination). One subject in the group given the 1:100 dilution did not have a vaccinial skin lesion but did have a virus-specific cytotoxic T-cell response after vaccination (Fig. 2A).

Enzyme-linked immunospot assays were used to determine the number of cells that produced vaccinia virus-specific interferon- $\gamma$ in peripheral-blood mononuclear cells obtained before and six months after vaccination. As shown in Figure 2B, the numbers were significantly different among the three groups. The presence of cells producing vaccinia virus-specific interferon- $\gamma$ correlated with the development of both a vesicle and a cytotoxic T-cell response. One subject who received the 1:100 dilution had a positive enzyme-linked immunospot assay and a cytotoxic T-cell response but did not have a skin response to the vaccine. Three subjects with vesicle formation did not have a positive enzyme-linked immunospot assay. In subjects with vesicle formation, the magnitude of the interferon- $\gamma$ response was significantly lower among those given the 1:10 dilution of vaccine or the 1:100 dilution than among those given the undiluted vaccine $(\mathrm{P}=0.038$ and $\mathrm{P}<0.001$, respectively).

\section{Lymphocyte Proliferation}

Lymphocyte proliferation was induced in response to vaccinia virus in 33 of 34 subjects with vesicle formation and in none of 23 subjects without vesicle formation (Fig. 2C). There were no significant doserelated differences in the stimulation index at six months among subjects with vesicle formation, al- 

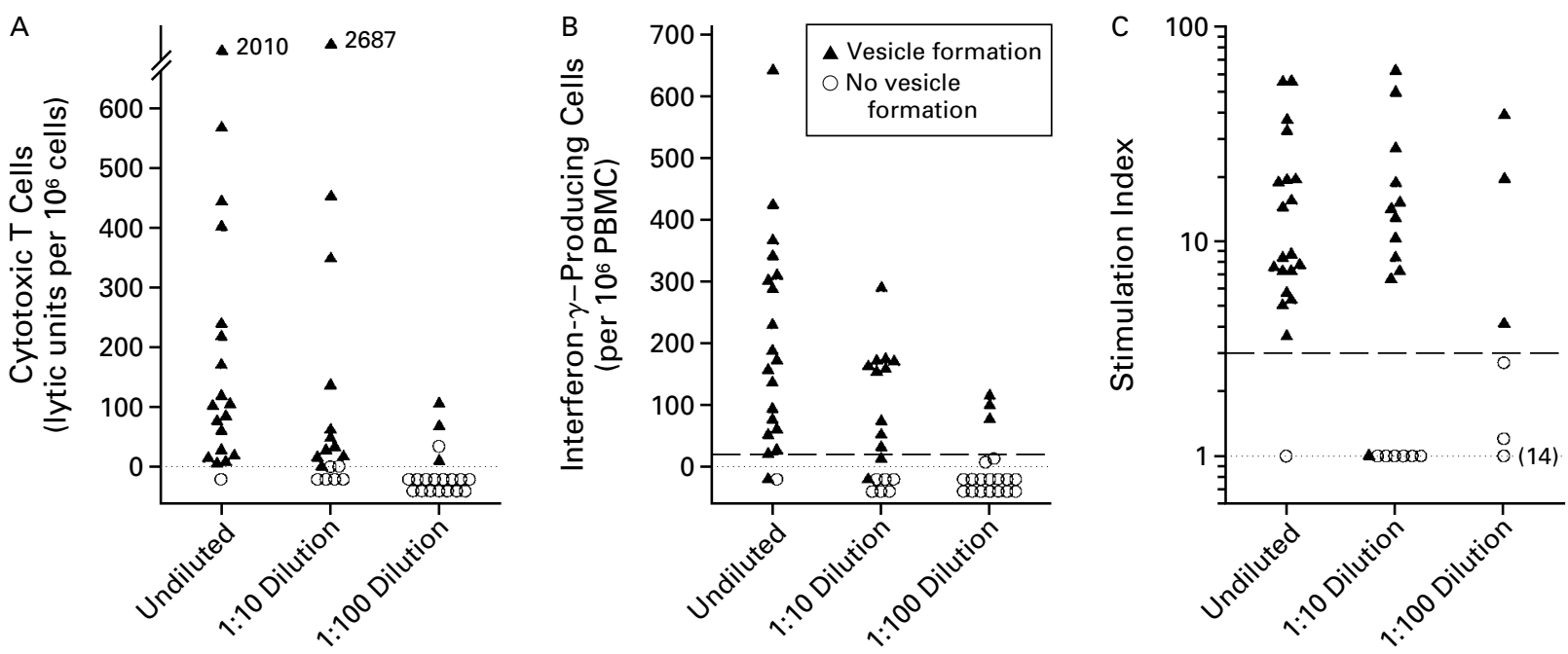

Figure 2. T-Cell Responses to Vaccinia Virus Six Months after Vaccination with Undiluted Vaccine, a 1:10 Dilution of Vaccine, or a 1:100 Dilution of Vaccine, According to the Presence or Absence of Vesicle Formation at the Inoculation Site within Seven to Nine Days after Vaccination.

Panel A shows the cytotoxic T-cell responses. The actual values for two outliers are shown. Panel B shows the number of cells producing vaccinia virus-specific interferon- $\gamma$. Panel $\mathrm{C}$ shows the degree of lymphocyte proliferation in response to vaccination. Two subjects with vesicles, one each from the group given undiluted vaccine and the group given the 1:10 dilution, had nonviable peripheral-blood mononuclear cells (PBMC) and could not be tested in any of the cell-mediated immune assays, and one subject in the group given the 1:100 dilution who did not have vesicle formation did not have blood drawn. One subject each in the group given undiluted vaccine and the group given the 1:10 dilution who had vesicle formation had high background values in the cytotoxic T-cell assay, which prevented interpretation of the results (Panel A). The stimulation index is the mean of the three intermediate values in each replicate of five. Higher values indicate greater proliferation of lymphocytes. The number in parentheses indicates the number of subjects with a stimulation index of 1, all of whom received the 1:100 dilution. Dashed lines in Panels B and C indicate the cutoff values for a positive response.

though there were significant overall dose-response differences in the stimulation index between the group given undiluted vaccine and the group given the 1:100 dilution $(\mathrm{P}=0.039)$, because of the higher frequency of nonresponse in the latter group. Examination of peripheral-blood mononuclear cells obtained before vaccination indicated that none of the subjects had had previous exposure to the virus (data not shown).

\section{DISCUSSION}

Immunization with vaccinia virus remains the only available option for protection against smallpox infection. The current supply of vaccine is viable and has a good titer, but dilution of the vaccine to titers of less than $10^{7}$ pfu per milliliter reduced the rates of successful vaccination. Serial dilution of the vaccine stock resulted in nonlinear reductions in the titer (e.g., a 1:100 dilution reduced the titer from $10^{7.8}$ pfu per milliliter to $10^{5.0}$ pfu per milliliter). This effect may result from the highly viscous nature of the preparation. As compared with the 95 percent rate of success associated with the undiluted vaccine, the rate of success associated with the dose of $10^{6.5} \mathrm{pfu}$ per milliliter provided by the 1:10 dilution was 70 percent. This lower rate is similar to the 75 percent rate reported among children who were vaccinated with a similar vaccine and dose $\left(10^{7}\right.$ pock-forming units per milliliter). ${ }^{19}$ The dose of $10^{5.0}$ pfu per milliliter provided by the 1:100 dilution was associated with a success rate of only 15 percent. These results indicate that serial dilution of the current vaccine to less than $10^{6.5}$ pfu per milliliter results in a loss of potency $(\mathrm{P}=0.001$ for the comparison of undiluted vaccine with a 1:10 dilution alone and $\mathrm{P}<0.001$ for the comparison of undiluted vaccine with both dilutions of vaccine).

There was a dose-response effect: higher doses produced significantly stronger cytotoxic T-cell and interferon- $\gamma$ responses. Given the small number of subjects in whom vaccination with a $1: 10$ or $1: 100$ dilution was successful, additional study is needed to confirm these observations. Findings in vaccinated children and adults with T-cell-related immunodeficiencies indicate that $\mathrm{T}$-cell responses are a critical element in the recovery from pox virus infections..$^{6-8}$ The cytotoxic T-cell and interferon- $\gamma$ responses in our study were much stronger than those reported after the receipt of experimental HIV-l vaccines and cor- 
respond to the levels of memory $T$ cells specific for measles virus in adults ${ }^{16}$ and to the strength of cytotoxic T-cell responses recorded in adults after the receipt of yellow fever vaccine. ${ }^{20}$ In addition, almost all subjects with vesicle formation had strong vaccinia virus-specific cytotoxic T-cell responses as well as increased numbers of interferon- $\gamma$-producing $\mathrm{T}$ cells. These findings suggest that, regardless of the dose of vaccine, if a vesicle forms, the resulting brisk $\mathrm{T}$-cell and humoral responses will be protective.

Diluting the available vaccine to titers of $10^{6.5} \mathrm{pfu}$ per milliliter or less reduced the frequency rates of local viral replication and vesicle formation - effects that are essential stimuli for protective immune responses, as evidenced by the lack of antibody, cytotoxic T-cell, and interferon- $\gamma$ responses. Previous reports in which smallpox vaccination in patients with defects in either cellular or humoral immunity led to severe vaccinia underscore the essential role of T-cell responses and antibody in protecting against pox viruses, such as variola. ${ }^{21}$ In our study, the induction of vaccinia virus-specific T-cell and B-cell responses was associated with clinically observable pox lesions. Previous studies have shown that the response is attenuated if the route of administration differs from the one that we used. ${ }^{19,22}$ We found that the absence of primary skin vesicles after the administration of diluted vaccine was associated with the absence of vaccinia virus-specific T-cell or B-cell responses and is therefore likely to indicate the absence of protective immunity. Future studies are needed to assess the effectiveness of vaccine titers of $10^{6.5}$ to $10^{8.0} \mathrm{pfu}$ per milliliter in both previously vaccinated and unvaccinated populations. Evaluation of new cell-culturederived vaccines should address both T-cell and B-cell responses. If vaccinations with dilutions of the current vaccine are administered, revaccination should be considered in persons without vesicle formation.

Supported by a contract (N01-AI-45250) with the National Institute of Allergy and Infectious Diseases.

We are indebted to James Meegan, Wendy Fanaroff-Ravick, and Catherine Laughlin at the National Institute of Allergy and Infectious Diseases, Bethesda, Md., and John Becher at the CDC for their assistance and thoughtful discussions, to Ruth Mazzeo for performing the proliferation assays, and to the staff at the Saint Louis Unipersity Vaccine and Treatment Evaluation Unit.

\section{REFERENCES}

1. Henderson DA. Smallpox: clinical and epidemiologic features. Emerg Infect Dis 1999;5:537-9.

2. World Health Organization. Declaration of global eradication of smallpox. Wkly Epidemiol Rec 1980;55:148.

3. Henderson DA. About the first National Symposium on Medical and Public Health Response to Bioterrorism. Emerg Infect Dis 1999;5:491.

4. Shalala D. Bioterrorism: how prepared are we? Emerg Infect Dis 1999; 5:492-3.

5. Russell PK. Vaccines in civilian defense against bioterrorism. Emerg Infect Dis 1999;5:531-3.

6. O'Connell CJ, Karzon DT, Barron AL, Plaut ME, Ali VM. Progressive vaccinia with normal antibodies: a case possibly due to deficient cellular immunity. Ann Intern Med 1964;60:282-9.

7. Fulginiti $\mathrm{V}$, Kempe $\mathrm{CH}$, Hathaway WE, et al. Progressive vaccinia in immunologically-deficient individuals. Birth Defects Orig Artic Ser 1968;5:129. 8. Redfield RR, Wright DC, James WD, Jones TS, Brown C, Burke DS. Disseminated vaccinia in a military recruit with human immunodeficiency virus (HIV) disease. N Engl J Med 1987;316:673-6.

9. Smallpox vaccine no longer available for civilians - United States. MMWR Morb Mortal Wkly Rep 1983;32:387.

10. Graham BS, Belshe RB, Clements $M L$, et al. Vaccination of vaccinianaive adults with human immunodeficiency virus type 1 gpl60 recombinant vaccinia virus in a blinded, controlled, randomized clinical trial. J Infect Dis 1992;166:244-52.

11. Katz JB. The effect of the virus-serum incubation period upon vaccinia virus serum neutralization titers. J Biol Stand 1987;15:389-92.

12. Iacono-Connors LC, Novak J, Rossi C, Mangiafico J, Ksiazek T. Enzyme-linked immunosorbent assay using a recombinant baculovirus-expressed Bacillus anthracis protective antigen (PA): measurement of human anti-PA antibodies. Clin Diagn Lab Immunol 1994;1:78-82.

13. Green $S$, Kurane I, Edelman R, et al. Dengue virus-specific human CD4+ T-lymphocyte responses in a recipient of an experimental live-attenuated dengue virus type 1 vaccine: bulk culture proliferation, clonal analysis, and precursor frequency determination. J Virol 1993;67:5962-7.

14. Demkowicz WE Jr, Littaua RA, Wang J, Ennis FA. Human cytotoxic

T-cell memory: long-lived responses to vaccinia virus. J Virol 1996;70: 2627-31.

15. Demkowicz WE Jr, Ennis FA. Vaccinia virus-specific CD8+ cytotoxic T lymphocytes in humans. J Virol 1993;67:1538-44.

16. Nanan R, Rauch A, Kampgen E, Niewiesk S, Kreth HW. A novel sensitive approach for frequency analysis of measles virus-specific memory T-lymphocytes in healthy adults with a childhood history of natural measles. J Gen Virol 2000;81:1313-9.

17. McClain DJ, Harrison S, Yeager CL, et al. Immunologic responses to vaccinia vaccines administered by different parenteral routes. J Infect Dis 1997; 175:756-63.

18. Holander M, Wolfe D. Nonparametric statistical methods. New York: John Wiley, 1973:115-6

19. Cherry JD, McIntosh K, Connor JD, et al. Clinical and serologic study of four smallpox vaccines comparing variations of dose and route of administration: primary percutaneous vaccination. J Infect Dis 1977;135:145-54. 20. Co MDT, Terajima M, Cruz J, et al. Human cytotoxic T lymphocyte responses to live attenuated $17 \mathrm{D}$ yellow fever vaccine: identification of HLA-B35-restricted CTL epitopes on nonstructural proteins NS1, NS2b, NS3 and the structural protein E. Virology 2002;243:151-63.

21. Lane JM, Ruben FL, Neff JM, Millar JD. Complications of smallpox vaccination, 1968: national surveillance in the United States. N Engl J Med 1969;281:1201-8

22. Connor JD, McIntosh K, Cherry JD, et al. Clinical and serologic study of four smallpox vaccines comparing variations of dose and route of administration: primary subcutaneous vaccination. J Infect Dis 1977;135:167-75.

Copyright (C) 2002 Massachusetts Medical Society. 